\title{
Tunneling Study of the Charge-Ordering Gap on the Surface of $\mathrm{La}_{0.350} \mathrm{Pr}_{0.275} \mathrm{Ca}_{0.375} \mathrm{MnO}_{3}$ Thin Films
}

\author{
Udai Raj Singh, S. Chaudhuri, Shyam K. Choudhary, R. C. Budhani, and Anjan K. Gupta \\ Department of Physics, Indian Institute of Technology Kanpur, Kanpur 208016, India.
}

(Dated: November 2, 2018)

\begin{abstract}
Variable temperature scanning tunneling microscopy/spectroscopy studies on (110) oriented epitaxial thin films of $\mathrm{La}_{0.350} \mathrm{Pr}_{0.275} \mathrm{Ca}_{0.375} \mathrm{MnO}_{3}$ are reported in the temperature range of 77 to 340 $\mathrm{K}$. The films, grown on lattice matched $\mathrm{NdGaO}_{3}$ substrates, show a hysteretic metal-insulator transition in resistivity at $170 \mathrm{~K}$. The topographic STM images show step-terrace morphology while the conductance images display a nearly homogeneous surface. The normalized conductance spectra at low temperatures $(\mathrm{T}<150 \mathrm{~K})$ show an energy gap of $0.5 \mathrm{eV}$ while for $\mathrm{T} \geq 180 \mathrm{~K}$ a gap of $0.16 \mathrm{eV}$ is found from the activated behavior of the zero bias conductance. The presence of energy gap and the absence of phase separation on the surface over more than $2 \mu \mathrm{m} \times 2 \mu \mathrm{m}$ area contradicts the metallic behavior seen in resistivity measurements at low temperatures. We discuss the measured energy gap in terms of the stabilization of the insulating $\mathrm{CO}$ phase at the film surface.
\end{abstract}

PACS numbers:

The research over past decade on colossal magnetoresistive (CMR) manganites [1, 2, 3] has led to a number of very interesting phenomena. These are now understood to be systems with strongly coupled charge, spin, lattice, and orbital degrees of freedom with a number of possible phases of nearly equal free energies [2, 4, ,5]. These phases are thought to coexist in some manganite compositions and the relative fractions of phase are sensitive to external perturbations such as magnetic field, electric field, pressure, and strain. In fact, a sudden termination of the periodic potential at the surface is a perturbation that seems to make the surface quite different from the bulk [6]. The bulk of some of the small band-width manganites [7] undergoes an electronic phase-separation [8, 9] into metallic and insulating regions, which is thought [4, 5, 10] to be responsible for the CMR effect. Various research groups have observed multiple phases in some of the manganites, in particular, in $\left(\mathrm{La}_{1-x} \mathrm{Pr}_{x}\right)_{0.625} \mathrm{Ca}_{0.375} \mathrm{MnO}_{3}$ series, using different probes [8, 11, 12] including Scanning Tunneling Microscopy / Spectroscopy (STM/S) 13, 14, 15, 16]. However, the detailed electronic nature of the individual phases is far from understood [2].

In this work, we have probed the surface of a relatively narrow band-width hole-doped manganite $\mathrm{La}_{0.350} \mathrm{Pr}_{0.275} \mathrm{Ca}_{0.375} \mathrm{MnO}_{3}$ (LPCMO), using the STM/S in the temperature range of $77 \mathrm{~K}$ to $340 \mathrm{~K}$. The ground state of the two end members of this compound $\mathrm{La}_{0.675} \mathrm{Ca}_{0.375} \mathrm{MnO}_{3}$ and $\mathrm{Pr}_{0.675} \mathrm{Ca}_{0.375} \mathrm{MnO}_{3}$ are ferromagnetic metal and antiferromagnetic charge-ordered (CO) insulator, respectively. Intermediate compositions show a CO transition around $200 \mathrm{~K}$ with coexisting $\mathrm{CO}$ insulating and charge-disordered conducting phases [8]. Our main objective in this study has been to probe various phases in LPCMO films by local tunneling spectroscopy to understand their nature and contribution to CMR. However, we find that the low energy part of the
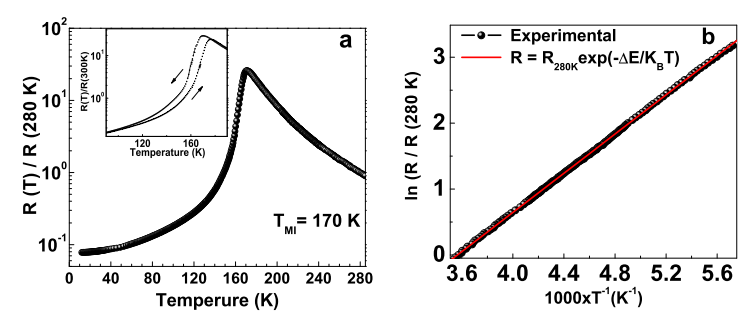

FIG. 1: a. Normalized resistance as a function of temperature showing the metal-insulator transition in LPCMO film at 170 $\mathrm{K}$. The inset in fig.1(a) showing the hysteric metal-insulator transition. The plot of $\ln \left(\mathrm{R} / \mathrm{R}_{280 K}\right)$ with $1000 / \mathrm{T}$ (for 280 $\mathrm{K}>\mathrm{T}>170 \mathrm{~K}$ ) is shown in the fig1.(b) with a linear fit giving an activation energy of $0.12 \mathrm{eV}$.

spectra evolves quite homogeneously with temperature with an energy gap that changes from $0.16 \mathrm{eV}$ to $0.5 \mathrm{eV}$ on cooling. Whilst the gap varies slightly on a nanometer length scale, it exists even below the insulator-metal transition temperature $\left(T_{M I}\right)$ measured by resistivity. This is the first temperature dependent STM/S study on this narrow bandwidth manganite, and our major finding is the persistence of a nearly homogeneous CO gap which we interpret in terms of the stabilization of the $\mathrm{CO}$ phase on the surface even though the bulk of the film shows a phase separated behavior.

Epitaxial films of thickness $\approx 200 \mathrm{~nm}$ of LPCMO were grown at a deposition rate of $\sim 0.3 \mathrm{~nm} / \mathrm{s}$ on (110) surface of $\mathrm{NdGaO}_{3}$ (NGO) single crystals by using pulsed laser deposition (PLD). The (110) direction refers to the orthorhombic unit cell of NGO with $\mathrm{d}_{110}=0.38595 \mathrm{~nm}$. A $\mathrm{KrF}$ excimer laser operated at $10 \mathrm{~Hz}$ with an areal energy density of $2 \mathrm{Jcm}^{-2} /$ pulse on the surface of a stoichiometric sintered target of LPCMO. For these films we find a step-terrace morphology for relatively slow growth conditions while the faster growth rates lead to a granular surface. In this paper, we focus on the STM/S study 
of LPCMO thin films with step-terrace surface morphology. Films with granular morphology have an associated electronic inhomogeneity and they may not reflect the film's intrinsic electronic behavior. For resistivity measurements, silver contact pads were deposited through a shadow mask in a four probe configuration. The STM/S measurements were performed using a homemade variable temperature STM with fresh cut $\mathrm{Pt}_{0.8} \mathrm{Ir}_{0.2}$ wire tip. The STM head is based on a design published elsewhere [17] while the electronics and software are commercial. The STM measurements reported here have been carried out in liquid nitrogen in 77 - $340 \mathrm{~K}$ temperature range in a cryogenic vacuum. After transferring the sample to the STM in air, the cryostat is pumped and flushed with He-gas a few times. The sample transfer is carried out quite fast so that the sample is exposed to air for less than $30 \mathrm{~min}$. Finally the cryostat is pumped to a high vacuum $\left(1 \times 10^{-4}\right.$ mbar $)$ prior to cooling in liquid nitrogen. To ensure a clean sample surface we heat the whole STM to $340 \mathrm{~K}$ for a few hours keeping the surroundings at liquid nitrogen temperatures.

The standard ac-modulation technique was used for STS and conductance spectra with a modulation amplitude between 30 and $50 \mathrm{mV}$. The dc-component of current, $I(V)$, is fed to the z-feedback for controlling and measuring the tip-sample separation while a lock-in amplifier measured the ac-component giving the conductance, dI/dV, at bias V. Simultaneous conductance and topographic images in constant-current mode at a bias $\left(\mathrm{V}=V_{0}\right)$ are obtained by measuring the lock-in output and the z-feedback voltage, respectively, over the surface. In this mode, the feedback, by varying tip-sample separation, ensures that the integrated conductance up to $V_{0}$ is the same at all points of the image. As a result, in the dark regions of a conductance image the current gets less contribution from the conductance at larger bias and more contribution from the small bias. Thus, in a conductance image, the darker regions represent more metallic (or less gapped) areas [18] and the topographic and conductance images are anti-correlated for flat surfaces.

The four probe resistance as a function of temperature for an LPCMO film (Fig:1a) shows an insulator-metal transition at $\mathrm{T}_{M I}=170 \mathrm{~K}$ with more than two orders of magnitude change in resistivity, which is typical of the narrow bandwidth CMR manganites [19]. The cooling and heating curves differ slightly near the transition indicating the presence of more than one phase in the bulk of this film as shown in the inset of Fig 1a. Resistivity in LPCMO single crystals and polycrystalline samples shows a small feature at $\sim 200 \mathrm{~K}$ due to opening of a $\mathrm{CO}$ gap [8, 12], which is absent in thin films [20, 21]. This could be due to the small substrate induced strain effect making the $\mathrm{CO}$ transition not so sharp with respect to temperature. The formation of charge ordered state below $210 \mathrm{~K}$ in the same composition thin films on NGO
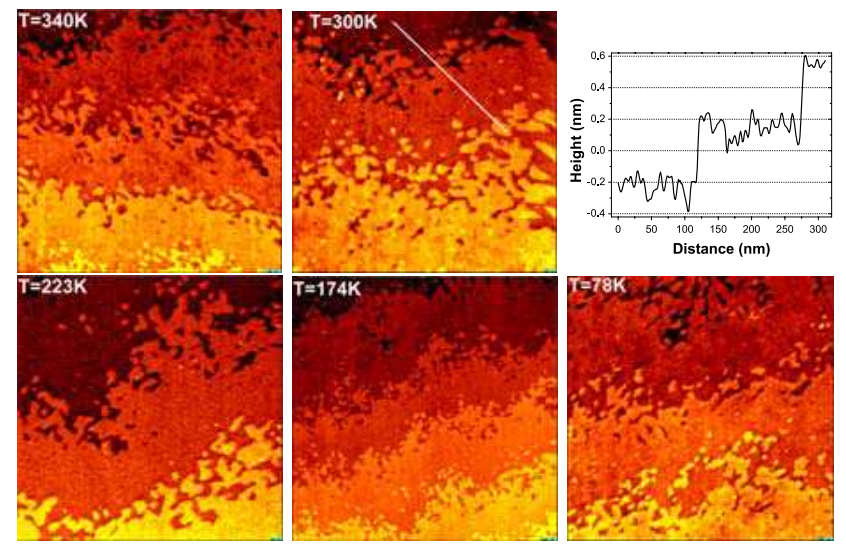

FIG. 2: Topographic images of LPCMO film at temperatures of $340 \mathrm{~K}$ (Scan size $=800 \times 800 \mathrm{~nm}^{2}$, Bias $=1 \mathrm{~V} /$ Current $=0.1 \mathrm{nA}), 300 \mathrm{~K}\left(502 \times 502 \mathrm{~nm}^{2}, 1.1 \mathrm{~V} / 0.1 \mathrm{nA}\right), 223 \mathrm{~K}$ $\left(504 \times 504 \mathrm{~nm}^{2}, 1.1 \mathrm{~V} / 0.1 \mathrm{nA}\right), 174 \mathrm{~K}\left(1.5 \times 1.5 \mu \mathrm{m}^{2}, 1.4 \mathrm{~V} /\right.$ $0.2 \mathrm{nA})$, and $78 \mathrm{~K}\left(700 \times 700 \mathrm{~nm}^{2}, 2.6 \mathrm{~V} / 0.3 \mathrm{nA}\right)$ The graph in the top right shows a line cut along the line marked in 300 $\mathrm{K}$ image with steps of height $0.4( \pm 0.05) \mathrm{nm}$.

substrate has been observed by electron microscopy and diffraction studies by Chaudhuri, et. al. 22]. In Fig 1], we plot $\ln \left(R / R_{280 K}\right)$ Vs $1 / \mathrm{T}$ for $170 \mathrm{~K}<\mathrm{T}<285 \mathrm{~K}$, which establishes the activated behavior of resistivity with an activation energy $(\Delta)$ of $0.12 \mathrm{eV}$. This is in close agreement with the average optical gap $(2 \Delta)$ of about $0.2 \mathrm{eV}$ in this temperature range observed by Lee et.al. [12] in LPCMO single crystals of slightly different composition.

The STM topographic images of the LPCMO film (in Fig(2) between 340 and $78 \mathrm{~K}$ temperature show stepterrace morphology with a terrace width of about 200 $\mathrm{nm}$. The rms roughness of each terrace is found to be less than $0.1 \mathrm{~nm}$. From the conductance images, as discussed later, some of this roughness can be attributed to the electronic inhomogeneities. The terrace boundaries are quite irregular together with a few isolated islands of lateral size up to ten nanometers. From the line-cut shown in Fig 2 , the height of the terrace is found to be $0.4( \pm 0.05) \mathrm{nm}$ in agreement with the step height of 0.38 $\mathrm{nm}$ for (110) planes of LPCMO. Further, a tiny $(0.2 \%)$ epitaxial mismatch between the NGO substrate and the LPCMO films for such large thickness would not leave significant strain near the surface. The presence of such smooth terraces allows local tunneling experiments in the regions that are unaffected by the presence of steps or grain boundaries and their associated local disorders and non-uniformities.

Fig 3 shows a set of simultaneously taken topographic and conductance images at $140 \mathrm{~K}$. As discussed earlier, the bright regions of a conductance image represent more gapped areas. The conductance images have some very bright spots of about $5 \mathrm{~nm}$ size with a relatively low coverage ( 20 spots $/ \mu m^{2}$ ). The image in Fig $3 \mathrm{~b}$ has a number of these bright spots. These spots are seen at 


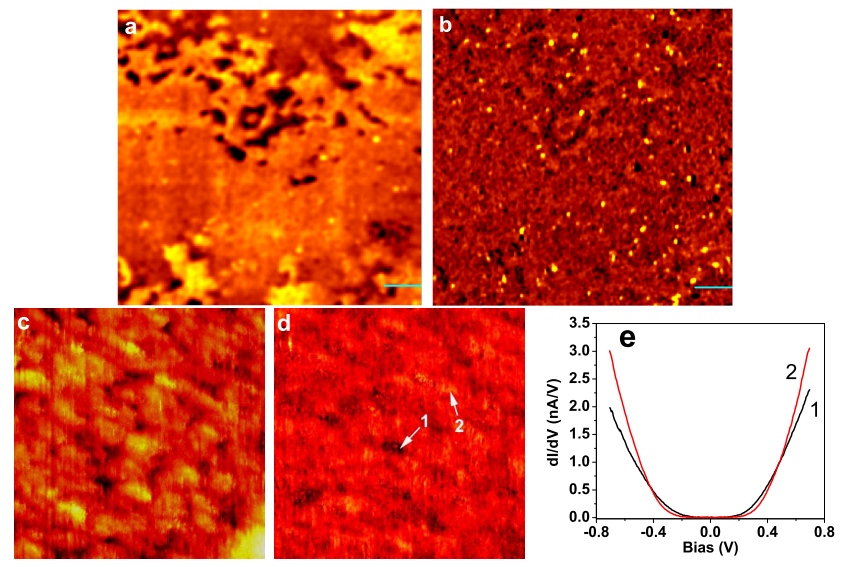

FIG. 3: Topographic (a and c) and corresponding conductance ( $\mathrm{b}$ and $\mathrm{d}$ ) images at $\mathrm{T}=140 \mathrm{~K}$ at bias $1.0 \mathrm{~V}$ and tunnel current value $0.1 \mathrm{nA}$. The size of images a and $\mathrm{b}$ is $416 \times 416$ $\mathrm{nm}^{2}$ and that of $\mathrm{c}$ and $\mathrm{d}$ is $46 \times 46 \mathrm{~nm}^{2}$. fig.(e) shows characteristic spectra at two representative points (dark and bright) of the image in $d$.

all temperatures and they have a large energy gap ( 1 to $1.5 \mathrm{eV}$ ) as found from the local spectra. It is possible that these are either second phase materials belonging to the parent compounds, which are all antiferromagnetic insulators, or oxides of the constituent metals. Since their fraction is very small it is difficult to detect them in Xray diffraction. Such defects are also not seen in high resolution TEM images of similar samples, which makes us believe that they are segregated at the surface.

Other than these large-gap-spots, a weak contrast in the conductance images (Fig $3 \mathrm{~d})$ is also seen at nanometer length scale, which is somewhat anti-correlated with the contrast of the simultaneous topographic image. The image in Fig $3 \mathrm{~d}$ is a smaller area image of region without any bright spots as discussed above. The crosscorrelation coefficient between the topographic and conductance images on terraces is found to be $\approx-0.2$ showing that the density of states (DOS) inhomogeneity is partly responsible for the topographic contrast. The detailed tunneling spectra, with two representative ones in Fig 3 , show that this inhomogeneity actually corresponds to a small variation in the CO-gap value. Similar inhomogeneity is seen at all temperatures from 77 to $340 \mathrm{~K}$. We believe that this electronic contrast represents some frozen chemical inhomogeneity in this non-stoichiometric compound.

A tunneling spectrum, other than the DOS, is also affected by finite temperatures and the voltage dependence of the tunneling matrix element [23, 24]. The temperature smears out the spectral features of width less than a few $k_{B} T$. The voltage dependence of the matrix element is more difficult to handle for large bias spectra as one does not know much about the details of the tunnel barrier. In general, one has to be careful in comparing the
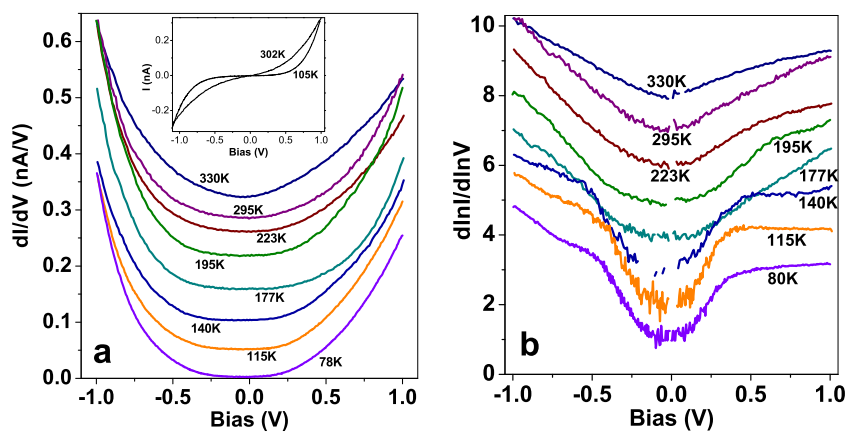

FIG. 4: Temperature dependent $\mathrm{dI} / \mathrm{dV}-\mathrm{V}$ (left) and $\mathrm{d} \ln \mathrm{I} / \mathrm{d} \ln \mathrm{V}-\mathrm{V}$ (right) spectra with junction bias $1.0 \mathrm{~V}$ and current $0.1 n \mathrm{~A}$. I-V spectra at two different temperature $302 \mathrm{~K}$ and $105 \mathrm{~K}$ are shown in the inset of the left plot. The consecutive spectra have been shifted upward for clarity.

absolute $\mathrm{dI} / \mathrm{dV}$ values at a given bias for two spectra that have different behavior with $\mathrm{V}$ as it is difficult to ensure the same tunneling matrix element for the two spectra. For such comparisons we have chosen the spectra with same junction resistances at a fixed bias. To extract the DOS from a $\mathrm{dI} / \mathrm{dV}$ spectra a widely used method [24] is to plot $(\mathrm{dI} / \mathrm{dV}) /(\mathrm{I} / \mathrm{V})($ or $\mathrm{d}(\operatorname{lnI}) / \mathrm{d}(\ln \mathrm{V}))$, which normalizes away the voltage dependence of the matrix element from the spectra. Practically, $d(\operatorname{lnI}) / d(\ln V)$ sharpens the spectral features that are not smeared away by temperature.

Temperature dependence of the tunneling spectra is shown in Fig 4 4 with each spectrum taken at a tunnel current set-point of $0.1 \mathrm{nA}$ at $1 \mathrm{~V}$ bias. The conductance near zero bias at low temperatures is nearly flat with a very small magnitude indicating the presence of a gap while that at higher temperatures has a noticeable curvature with significant conductance value. This indicates opening of an energy gap with cooling. The low temperature gap is more clearly visible in $\mathrm{d} \ln \mathrm{I} / \mathrm{d} \ln \mathrm{V}-\mathrm{V}$ plots in Fig 40. We also show two representative I-V spectra in the inset of Fig 4a. The gap value $(2 \Delta)$ at low temperatures is found to be $0.5( \pm 0.1) \mathrm{eV}$ from the separation between the center of the two gap edges in $\mathrm{d} \ln \mathrm{I} / \mathrm{d} \ln \mathrm{V}$ spectra (Fig 4 ). This is in reasonable agreement with the low temperature gap of $0.38 \mathrm{eV}$ found in optical measurements of Lee et.al. [12]. As mentioned earlier, this gap value has small $(\approx 0.1 \mathrm{eV})$ variations over a few nanometer length scale but the spectra are qualitatively homogeneous. To look for the phase separation, we have studied the spectra over reasonably large areas $(>2 \mu \mathrm{m} \times 2 \mu \mathrm{m})$ with step-terrace morphology without any signature of non-gapped spectra at low temperatures. We have also thermally cycled this sample several times but this behavior remains unchanged. At higher temperatures, the zero bias conductance (ZBC), $\sigma_{0}$, is found to show an activated behavior indicative of the presence of a smaller gap that is thermally smeared out. 


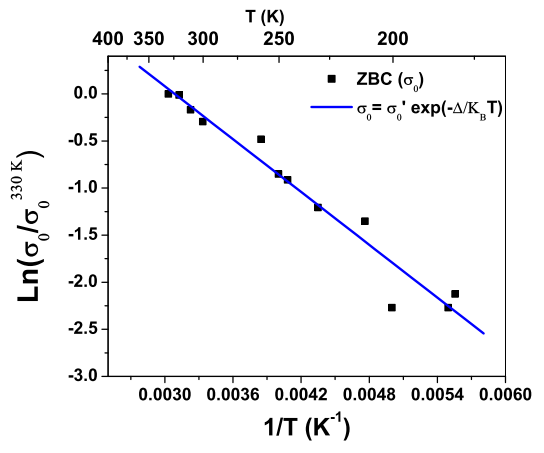

FIG. 5: $\ln \left(\sigma_{0}\right)$ vs $1 / \mathrm{T}$ showing an activated behavior of the ZBC with an energy gap $(\Delta)$ of $0.08 \mathrm{eV}$. The temperature scale is shown on top of $\mathrm{X}$-axis.

$\operatorname{Ln}\left(\sigma_{0}\right)$ above $180 \mathrm{~K}$ is plotted in Fig 5 with respect to $1 / \mathrm{T}$. The linear fit shown in the same figure gives an activation gap $(\Delta)$ of $0.08 \mathrm{eV}$.

Phase separation into a charge ordered insulating and a charge disordered conducting phase on sub-micron scale is well established in LPCMO bulk as well as thin films. This is also apparent in thin films studied here from the hysteretic behavior of resistivity. The charge-order exists in LPCMO both below and above $T_{M I}[25]$ with a change in the $\mathrm{CO}$ fraction with temperature and magnetic field [8]. The insulating behavior above $T_{M I}$ of the LPCMO films studied here can be attributed to the presence of the $\mathrm{CO}$ phase. We found an energy gap $(\Delta)$ above $T_{M I}$ of magnitude $0.12 \mathrm{eV}$ from the resistivity measurements while the gap found from the ZBC of the tunneling spectra is $0.08 \mathrm{eV}$. These two are consistent with each other given that one probes the surface while the other probes the bulk of the film. An energy gap $(2 \Delta)$ of $0.5 \mathrm{eV}$ below $T_{M I}$ is found from $\mathrm{dlnI} / \mathrm{dln} V$ spectra. Both these gaps agree reasonably well with those observed by optical spectroscopy [12].

The tunneling measurements above $T_{M I}$ agree with the bulk resistivity but the two show quite opposite behavior at low temperatures. It is impossible to get such conducting behavior in bulk at low temperatures with such a pronounced gap in the electronic DOS in this three dimensional compound. This result is extremely puzzling and the only reconciliation we can think of is that the tunneling somehow is not probing the bulk properties of the film. Surface contamination is the first thing to blame and we cannot rule this out completely as we do expose our samples to air but for a very short time while transferring them from the growth chamber to the STM. However, excellent terraces over large areas and a good correlation of the temperature dependent tunneling data with charge-ordering makes us strongly believe that we are probing intrinsic properties of the surface of the films, which has a charge-ordered phase stabilized.

The X-ray spectroscopy and spin polarized tunneling studies on manganites [6] also show that the surface layer is insulating with significant suppression in ferromagnetism. The surface is also found to be different from the bulk from the NMR studies on polycrystalline manganites 26]. In LPCMO, this effect could be quite strong as it is a three dimensional compound. So it is reasonable to assert that a sudden termination of the lattice stabilizes a charge-ordered insulating phase on the surface. Surface reconstruction or a different chemical composition of the film, particularly oxygen stoichiometry, near the surface are the two possibilities that we can speculate. Nevertheless, this seems to be an intrinsic feature of manganites and it will play a significant role in polycrystalline samples [27, 28] and devices utilizing the spin-polarization 29] of the surface electrons.

In conclusion, our STM/S studies on epitaxial $\mathrm{La}_{0.350} \mathrm{Pr}_{0.275} \mathrm{Ca}_{0.375} \mathrm{MnO}_{3}$ films on $\mathrm{NdGaO}_{3}$ substrate show a temperature dependent energy gap. Conductance images show some inhomogeneity on nanometer length scale; however, no sign of any metallic phase was found in the tunneling spectra for $\mathrm{T}<\mathrm{T}_{M I}$. The low temperature gap seen here is inconsistent with the resistivity measurements which shows a metallic behavior below $\mathrm{T}_{M I}$. From the correlation of the energy gap with the CO phase we conclude that a charge-ordered insulating phase remains stabilized near the surface, which masks the signatures of the phase separation in the bulk of the film.

\section{ACKNOWLEDGEMENTS}

URS acknowledges financial support from CSIR, AKG acknowledges a research grant from DST of the Govt. of India. S. Rajauria's contribution to the initial work on the STM set-up is acknowledged.

[1] CMR, Charge Ordering and Related Properties of Manganese Oxides, ed. by C. N. R. Rao and B. Raveau (World Scientific, Singapore, 1998).

[2] M. B. Salamon and M. Jaime, Rev. Mod. Phys. 73, 583 (2001); M. Imada, A. Fujimori, and Y. Tokura, Rev. Mod. Phys. 70, 1039 (1998).

[3] R. von Helmolt, J. Wecker, B. Holzapfel, L. Schultz, and K. Samwer, Phys. Rev. Lett. 71, 2331 (1993);

[4] E. Dagotto, T. Hotta, and A. Moreo, Phys. Rep. 344, 1 (2001).

[5] K. H. Ahn T. Lookman, and A. R. Bishop, Nature 428, 401 (2004).

[6] H. Dulli, P. A. Dowben, S.-H. Liou, and E. W. Plummer, Phys. Rev. B 62, R14629 (2000); J. W. Freeland, K. E. Gray, L. Ozyuzer, P. Berghuis, E. Badica, J. Kavich, H. Zheng, and J. F. Mitchell, Nature Materials 4, 62 (2005).

[7] H. Y. Hwang, S-W. Cheong, P. G. Radaelli, M. Marezio, and B. Batlogg, Phys. Rev. Lett. 75, 914 (1995).

[8] M. Uehara, S. Mori, C. H. Chen, and S.-W. Cheong, Nature 399, 560 (1999). 
[9] K. H. Kim, M. Uehara, C. Hess, P. A. Sharma, and S-W. Cheong, Phys. Rev. Lett. 84, 2961 (2000).

[10] A. Moreo, S. Yunoki, and E. Dagotto, Science 283, 2034 (1999).

[11] L. Zhang, C. Israel, A. Biswas, R. L. Greene, and A. de Lozanne, Science 298, 805 (2002).

[12] H. J. Lee, K. H. Kim, M. W. Kim, T. W. Noh, B. G. Kim, T. Y. Koo, S.-W. Cheong, Y. J. Wang, and X. Wei, Phys. Rev. B 65, 115118 (2002).

[13] M. Fäth S. Freisem, A. A. Menovsky, Y. Tomioka, J. Aarts, and J. A. Mydosh, Science 285, 1540 (1999).

[14] V. Moshnyaga, L. Sudheendra, O. I. Lebedev, S. A. Köster, K. Gehrke, O. Shapoval, A. Belenchuk, B. Damaschke, G. van Tendeloo, and K. Samwer, Phys. Rev. Lett. 97, 107205 (2006).

[15] Ch. Renner, G. Aeppli, B.-G. Kim, Y.-A. Soh, and S.W. Cheong, Nature 416, 518 (2002); H. M. Rønnow, Ch. Renner, G. Aeppli, T. Kimura and Y. Tokura, Nature 440, 1025 (2006).

[16] T. Becker, C. Streng, Y. Luo, V. Moshnyaga, B. Damaschke, N. Shannon, and K. Samwer, Phys. Rev. Lett. 89, 237203 (2002).

[17] A. K. Gupta and K.-W. Ng, Rev. Sci. Instrum. 72, 3552 (2001).

[18] Ch. Renner and H. M. Rønnow, chapter II.9 in Scanning Probe Microscopy: Electromechanical Phenomena at the Nanoscale, Springer Series (2006).

[19] T. Wu, S. B. Ogale, S. R. Shinde, A. Biswas, T. Polletto,
R. L. Greene, T. Venkatesan, and A. J. Millis, J. Appl. Phys. 93, 5507 (2003).

[20] N. K. Pandey, R. P. S. M. Lobo, and R. C. Budhani, Phys. Rev. B 67, 054413 (2003); T. Dhakal, J. Tosado, and A. Biswas, Phys. Rev. B 75, 092404 (2007).

[21] W. Prellier, A. Biswas, M. Rajeswari, T. Venkatesan, and R. L. Greene, Appl. Phys. Lett. 75, 397 (1999).

[22] S. Chaudhuri, R. C. Budhani, J. He, and Y. Zhu, Phys. Rev. B 76, 132402 (2007).

[23] E. L. Wolf, Principles of Electron Tunneling Spectroscopy, Oxford Univ. Press, New York, (1989).

[24] J. A. Stroscio, R. M. Feenstra, and A. P. Fein, Phys. Rev. Lett. 57, 2579 (1986).

[25] V. Kiryukhin, B. G. Kim, V. Podzorov, S.-W. Cheong, T. Y. Koo, J. P. Hill, I. Moon and Y. H. Jeong, Phys. Rev. B 63, 024420 (2000).

[26] M. Bibes, Ll. Balcells, J. Fontcuberta, M. Wojcik, S. Nadolski, and E. Jedryka, Appl. Phys. Lett. 82, 928 (2002).

[27] H. Y. Hwang, S-W. Cheong, N. P. Ong, and B. Batlogg, Phys. Rev. Lett. 77, 2041 (1996).

[28] L. Sudheendra and C.N.R. Rao, J. Phys.: Cond, Matt. 15, 3029 (2003).

[29] F. Pailloux, D. Imhoff, T. Sikora, A. Barthélémy, J.-L. Maurice, J.-P. Contour, C. Colliex, and A. Fert, Phys. Rev. B 66, 014417 (2002). 